Questions de communication

$24 \mid 2013$

Renouvellement des mises en scène télévisuelles de la politique

\title{
Paul JORION, La guerre civile numérique
}

Paris, Textuel, coll. Conversations pour demain, 2011, 109 pages

\section{Gilles Boenisch}

\section{OpenEdition}

\section{Journals}

Édition électronique

URL : http://journals.openedition.org/questionsdecommunication/8844

DOI : 10.4000/questionsdecommunication. 8844

ISSN : 2259-8901

\section{Éditeur}

Presses universitaires de Lorraine

Édition imprimée

Date de publication : 31 décembre 2013

Pagination : 318-320

ISBN : 978-2-8143-0182-5

ISSN : 1633-5961

\section{Référence électronique}

Gilles Boenisch, «Paul JoRıon, La guerre civile numérique », Questions de communication [En ligne], 24 |

2013, mis en ligne le 01 février 2014, consulté le 22 septembre 2020. URL : http://

journals.openedition.org/questionsdecommunication/8844 ; DOl : https://doi.org/10.4000/

questionsdecommunication. 8844 
universalité des outils [...], universalité géographique $[\ldots]$, universalité des rôles [...], universalités des savoirs. L'apparition d'une autre culture, réellement globale, résulte de ces universalités $\gg($ p. $|3|)$. Là réside peut-être la clé des conditions numériques. Soit les données restent un bien privé à la discrétion exclusive des géants de l'internet, soit elles pourraient être un bien commun universel.

Finalement, l'essai tente de fournir une vulgarisation de la prégnance des activités numériques. La réflexion s'efforce de se construire sur l'humain conceptualisé dans les technologies de l'information et de la communication, à partir d'une succession de modules textuels. Cette construction atypique explique peut-être les fréquentes et parfois étranges références, indiquant « d'évidence » que l'homme a toujours été indissociable de ses activités sociales et techniques. En cela, à défaut de présenter une réelle problématique, La condition numérique illustre ses propos avec des citations d'auteurs antérieurs au numérique : Marshall McLuhan, William Gibson, Pierre Teilhard de Chardin, Jean Baudrillard, Vladimir Nabokov, Arthur C. Clarke, Jules Verne, Karl Marx, André Malraux, Alexis de Tocqueville... Pourtant, même si cette mise en perspective décalée a le mérite d'ouvrir un interstice dialogique, elle n'arrive pas à éclairer suffisamment les thématiques successives pour que l'on puisse aller au-delà d'un discours grand public. On aurait aimé que les textes incitent à investir plus que l'immédiateté et parfois, la superficialité des propos, en se donnant les moyens d'une analyse pluridisciplinaire prenant en compte, a minima, les écrits spécialisés et les enjeux s'y attenant.

Néanmoins, l'ouvrage suscite l'intérêt pour ce qu'il livre des auteurs : un instantané des conceptualisations et des visions d'auteurs référents au cœur des sphères médiatiques privées et publiques, décrivant ce quilis perçoivent de l'univers numérique qu'lls s'efforcent « d'exploiter ».

Gilles Boenisch

CREM, université de Lorraine, F-57000 gilles.boenisch@gmail.com

\section{Paul JORION, La guerre civile numérique.}

Paris, Éd. Textuel, coll. Conversations pour demain, 201I, 109 p.

Reposant sur un travail de notes de terrain au sens anthropologique, l'essai de Paul Jorion recroise les données de son blog très populaire sur l'internet. Le chercheur avait été l'un des premiers à prévoir la crise des subprimes et la récession qui allait en résulter. Spécialisé à la fois en économie, en intelligence artificielle et en anthropologie sociale, Paul Jorion conduit une réflexion informée et hétérodoxe sur les nouvelles formes de critique sociale et d'activisme politique qui se développent via l'internet. Dans La guerre civile numérique, il revient sur l'épisode Wikileaks et les réactions politiques qui l'ont accompagné. il dépeint une situation d'extrême tension qu'il qualifie de " prérévolutionnaire », qui ne serait pas un mouvement émanant du bas pour s'élever contre les pouvoirs en place. À l'inverse, il s'agirait d'« une déclaration de guerre » qui vient des gouvernements, contre les mouvements citoyens.

En quoi Julian Assange représente-t-il une menace pour de nombreux gouvernements ? Les réseaux sociaux favorisent-ils l'apparition de révoltes populaires? Comment les hackers contournent-ils les mécanismes de surveillance généralisée mis en œuvre par les gouvernements? Peut-on « éteindre » l'internet, comme le prévoient les États-Unis (Loi Kill-Switch, p. 72) pour empêcher une révolution? Existe-t-il des seuils psychologiques qui, une fois franchis, rendent les événements futurs totalement indécidables? Les blogueurs peuvent-ils concurrencer les journalistes? Autant de problématiques pour lesquelles Paul Jorion donne des éléments d'analyse dans l'ouvrage.

La première partie, « Les hacktivistes : cyber-terroristes ou dissidents numériques? 》 (pp. 10-47), dénonce la collusion profonde entre les gouvernements et les entreprises privées, faisant conjointement appel à des officines privées de sécurité pour contenir les « déviants 》. Qualifiés par le gouvernement américain de « terroristes high-tech », de « combattants ennemis à neutraliser » (pp. 19-22), les dissidents, hackers et Anonymous, se présentent comme des agents subversifs au service de la transparence démocratique, une transparence institutionnelle refusée au nom de la nécessité des niveaux dintervention officiel et officieux. En réalité, les gouvernements n'arrivent pas à s'accommoder de la transparence. Finalement, la démocratie n'est qu'un discours de façade, une image. En contrepartie, «le fait qu'lnternet offre un relatif anonymat a toujours été considéré comme un danger par les autorités » (pp. 25-26). En ce sens, l'intervention majeure de Wikileaks a prouvé « que les entreprises s'approprient activement l'autorité de l'État pour assurer et augmenter leur propre pouvoir. L'exemption de l'État de droit a été purement et simplement transférée du sommet des élites politiques à leurs contreparties du secteur privé » (p. 38). À cette occasion, on découvrait que les États s'adressaient « à des agences s'occupant en principe de sécurité, mais en réalité essentiellement de 
renseignement, de désinformation et de sabotage, pour tenter de ternir la réputation de ceux quills considéraient comme [leurs] adversaires ». Pour préserver un « écosystème de corruption 》 (pp. 38-39), les États emploient « des méthodes mises au point pour contrer une éventuelle menace terroriste [...] à l'encontre des citoyens ordinaires » (p. 40). En cela, les informations divulguées ont un réel potentiel réformateur : « Les gens qui les connaissent l'ont bien compris [...]. Ils s'emploient activement à empêcher cette réforme... 》); l'organisation et la publication de ces informations constitue donc « une action intrinsèquement antiautoritaire $\gg($ p. 46).

Ceque décritPaul Jorionn'est pas l'émergence spontanée d'une insurrection numérique, puisquili s'agit d'abord de mesures antidémocratiques prises par les États, posant les jalons « d'une guerre civile » que les gouvernements auraient « déjà perdue ». Si l'organisation de l'internet se prête parfaitement à une nouvelle forme de résistance contre l'arbitraire des pouvoirs traditionnels, « pour ces pouvoirs, c'est incontestablement une guerre perdue d'avance. Ils pourront mettre en place les mesures de contrôle quills veulent, ils ne gagneront pas... », car l'internet « interprète la censure comme une avarie et la contourne » comme en témoigne John Perry Barlow, célèbre défenseur des droits et de la liberté du numérique et auteur de la Déclaration dindépendance du Cyberspace (p. 32, 1996, accès : https://projects.eff.org/ barlow/Declaration-Final.html, consulté le 03/10/13). On l'aura compris, dans cette partie, Paul Jorion dissèque autant les concepts que les processus utilisés dans l'affrontement de deux camps diamétralement opposés. En réalité, cette notion de « guerre civile » relate la mise en jeu de la pérennité du système capitaliste actuel.

La seconde partie, « Révoltes et situations prérévolutionnaires à l'heure d'Internet » (pp. 48-90), s'attache à décrire les mécanismes de la crise récente, dans une perspective économique et psychologique. Paul Jorion y décrypte un stade « prérévolutionnaire » caractérisé par l'instabilité du système sociopolitique et financier, accompagné par la perte de confiance des populations dans leur gouvernance. À cela s'ajoute les potentialités de l'internet, permettant de diffuser rapidement les idées, de communiquer et de rendre lisible, de révéler. Le contrôle est devenu plus complexe et les mouvements « bien moins prévisibles qu'auparavant, ce qui explique la soudaineté des révolutions récentes » et l'impuissance des autorités y faisant face (p. 5). En situation prérévolutionnaire, le mécontentement et le sentiment d'injustice se développent.
« Le consensus de l'ordre existant a disparu. C'est ce que les sondages révèlent, c'est ce que révèlent aussi les élections : l'abstention est massive dans le monde occidental, et la majorité de ceux qui votent émettent un vote de protestation : ils ne votent pas pour des représentants spécifiques, ils votent, élection après élection, contre ceux qui étaient au pouvoir. L'usage du terme "prérévolutionnaire" pour désigner la situation actuelle ne me semble donc pas particulièrement aventureux » (pp. 53-54).

Aussi s'ajoute une série continue d'événements qui « démontrent l'existence de solidarités de caste, indépendamment des nationalités, et ces solidarités dessinent du coup les lignes d'une contagion révolutionnaire éventuelle 》. «Si les démocraties fonctionnent toujours au niveau des apparences, elles ne le font plus dans les faits » (p. 55). Pourtant, si les populations se scandalisent plus qu'auparavant, c'est parce « l'esprit de chacun est en alerte » (pp. 55-56), nourrissant le réseau d'un « cerveau collectif » (p. 75) au sein duquel les idées les plus convaincantes se détachent des autres et apparaissent au grand jour. C'est là que résident les potentialités du réseau où, malgré l'existence de processus de contrôle, persistent des interstices dans lesquels des formes de résistance collective peuvent s'élaborer : « Le contrôle augmente sans cesse, mais aussi les techniques pour déjouer ces contrôles. De toute façon, le "bruit" généralisé sur le web fait qu'on ne peut pas tout contrôler » (p. 7I). Dans ce phénomène, l'auteur entrevoit un moyen d'amplification de la perturbation des systèmes établis, une « rétroaction positive » débouchant vers un éclatement, une destruction conduisant au changement.

Dans la dernière partie, « Les blogs, entre subversion et information »(pp. 91-104), Paul Jorion relate sa propre expérience de création de blog, le blog portant sur l'économie le plus lu selon le classement Wikio (accès : http://www.pauljorion.com/blog/?p=23904, consulté le 03/10/13). Le chercheur pointe les possibilités critiques des publications indépendantes et personnelles sur l'internet et l'opportunité qui permettrait à des auteurs ou des communautés de se situer à la pointe de l'information sur des sujets desquels ils sont spécialistes. Cette formule semble plus en adéquation avec le « journalisme d'investigation » que celle exploitée par la presse traditionnelle fondée sur des « mécanismes de cooptation, qui imposent des filtres, et qui finissent par ne produire que des systèmes de pensée qui dégénèrent rapidement en raison d'une consanguinité intellectuelle trop élevée » (p. 93). À l'inverse, sur l'internet, « l'information circule très rapidement et de manière non maîtrisable » 
(p. 98), loin de l'autocensure journalistique. Finalement, avec La guerre civile numérique, Paul Jorion offre une analyse inédite des nouvelles formes de résistance numérique, à partir de réflexions parfois provocantes, inattendues, toujours au plus près du terrain.

Gilles Boenisch

CREM, université de Lorraine, F-57000 gilles.boenisch@gmail.com

\begin{abstract}
Jane McGonigal, Reality is Broken. Why Games make us Better and How They can change the World [La réalité est cassée. Pourquoi les jeux nous rendent meilleurs et comment ils peuvent changer le monde]. Londres, Penguin Books, 201 I, 400 pages.
\end{abstract}

Jane McGonigal a inventé le terme « jeu à réalité alternée » (《 alternate reality game ») pour désigner des jeux qui alternent jeu en ligne et action dans le monde tangible (p. I20), « un drame interactif qui se joue à la fois en ligne et dans le monde réel » (Henry Jenkins, La culture de la convergence: des médias au transmédia, Paris, A. Colin, 2013, p. 161). Reality is Broken s'ouvre sur le constat que les sociétés offrent peu d'occasions de se faire plaisir, contrairement à l'univers du jeu. Pour cette raison, l'auteure conclut son introduction par ces mots : «la réalité, comparée aux jeux, est cassée » (p. 3).

Parmi les originalités de l'ouvrage, l'auteure prend à contrepied la critique principale adressée au jeu selon laquelle celui-ci serait une fuite. Et si, au contraire, les jeux permettaient de changer les modes de pensée et les façons d'agir dans la vie quotidienne? Dans un premier temps, ane McGonigal propose une définition du jeu détachée de sa réception (" playing ») qui, elle, sera l'objet de la première partie de l'ouvrage intitulée « Pourquoi les jeux nous rendent heureux 》 (pp. 17-I I5). Selon la spécialiste des jeux vidéo, le jeu « se définit par son but, ses règles, son système de feedback, et la participation volontaire du joueur » (p. 21). Reprenant les commentaires de Mihály Csikszentmihályi (Beyond Boredom and Anxiety : The Experience of Play in Work and Games, San Francisco, Jossey-Bass, 1975), elle explique que jouer à un bon jeu peut éveiller une expérience optimale chez le joueur. L'état de flot ou l'expérience optimale est une absorption mentale accompagnant les moments de bonheur, ce sentiment se caractérisant par une résorption du temps, une grande impression de liberté, un sentiment de joie et d'accomplissement personnel. Mais, pour connaître l'expérience optimale, le joueur ne doit pas jouer pour gagner, l'expérience doit rester autotélique. L'expérience optimale devient un argument central pour l'auteure qui défend l'idée d'une généralisation du jeu dans toutes les dimensions de la vie. Elle cite la méthode ESM (experience sampling method) utilisée en psychologie - notamment par Mihaly Csikszentmihalyi (ibid.) -, pour affirmer que se détendre devant la télévision ou en faisant du lèche-vitrine ne fait que reporter notre état dépressif à plus tard. C'est ce que Jane McGonigal nomme le divertissement relaxant. Au contraire, les jeux en ligne sont des divertissements « durs 》 (《hard fun ») qui proposent une expérience de stress positif, un eustress, « eu » signifiant en grec « se sentir bien 》. L'expérience du hard fun éveillerait une émotion forte et valorisante, à savoir un sentiment spécifique de fierté (《f fiero ») dû à la victoire sur un adversaire (p. 33). Pour l'auteure, il s'agit de l'une des expériences les plus fortes en termes neurochimiques car cette victoire sur l'adversaire impliquerait trois circuits de récompense dans le cerveau. Ainsi, tant d'un point de vue social que physiologique, les jeux amélioreraientils le quotidien. Pour nous en convaincre, l'auteure décrit l'un de ses jeux favoris pratiqué en famille, Chore Wars, un jeu à réalité alternée. Chore Wars est une version simplifiée et gratuite de World of Warcraft à la différence que chacune des quêtes proposées en ligne correspond à une tâche ménagère dans le monde réel. Ce jeu se joue entre colocataires, en famille ou au bureau et non avec des étrangers ou des amis éloignés les uns des autres. Chaque joueur crée un avatar en ligne pour assumer une mission et participer à l'aventure. Ces missions sont entrées dans une base de données personnalisée. Celle de l'auteure comprend : vider le lave-vaisselle, faire une lessive, faire les sanitaires, etc. À chaque corvée effectuée, le joueur gagne des points. Selon elle, l'intérêt de ce jeu est que le résultat de l'action est visible, il existe un réel feedback ou retour d'information de l'action engagée. Cela se perçoit notamment par l'amélioration de l'apparence de l'avatar et par le fait que les victoires sont accomplies sous le regard des concurrents. La dimension collective du jeu serait donc la clé de son succès et sa vertu serait d'apprendre le travail collectif, non soumis à la contrainte puisque la corvée a été délibérément choisie.

Selon Jane McGonigal, il existe un fossé grandissant entre les environnements numériques et la salle de cours. Pour l'auteure, l'école idéale est une école dans laquelle on joue du matin jusqu'au soir, dans chaque cours, chaque activité (p. 127). Une telle école a ouvert ses portes à New York en 2009, après deux années de préparation : il s'agit de l'école publique Quest to Learn qui n'a pu voir le jour que grâce aux financements des fondations McArthur et Bill 